Original Paper http://ajol.info/index.php/ijbcs http://indexmedicus.afro.who.int

\title{
Effets du chaponnage sur les performances zootechniques du poulet local dans la ville de Ngaoundéré au Cameroun
}

\author{
S.A. MEBANGA*, I. GAPELBE et J.P. MINGOAS \\ Ecole des Sciences et de Médecine Vétérinaire - Département de Production Animale Université de \\ Ngaoundéré B.P: 268 Ngaoundéré - Cameroun. \\ *Auteur correspondant ; E-mail: mebengasaaristide@gmail.com
}

\section{RESUME}

Le chaponnage est une opération qui consiste à castrer et à engraisser un poulet (coquelet) afin d'atteindre une plus grande masse, une plus grande tendreté et donc d'améliorer la qualité de sa viande. Le coquelet castré est appelé chapon : c'est la volaille des fêtes sous d'autres cieux. A travers la création de valeur ajoutée sur des sujets considérés comme des non-valeurs économiques, le chaponnage peut contribuer à l'essor de l'aviculture camerounaise. En vue d'évaluer les effets du chaponnage sur les performances zootechniques du poulet local (villageois), une étude a été réalisée du $1^{\mathrm{er}}$ avril au 17 novembre 2018 dans la zone périurbaine de la ville de Ngaoundéré. Elle a porté sur 25 animaux répartis en deux lots et constitués à partir des parentaux acquis auprès des éleveurs locaux. Le lot 1 était constitué de 14 sujets ayant subi le chaponnage et le lot 2 formé de 11 mâles entiers. Le chaponnage a été réalisé à 8 semaines d'âge et les deux lots ont reçu une alimentation ad libitum. L'analyse statistique a montré que le gain de poids et l'indice de consommation ont varié de manière significative $(\mathrm{p}<0,05)$ chez les chapons par rapport aux coquelets entiers. Il en est de même en ce qui concerne le rendement carcasse et le poids des viscères. Les poids du cœur et du foie sont significativement $(p<0,05)$ inférieurs chez les chapons tandis que le poids du gésier est significativement $(\mathrm{p}<0,05)$ supérieur chez les chapons par rapport aux mâles entiers. L'efficacité alimentaire a diminué avec l'âge dans les deux lots. Le gras abdominal qui était de $40,18 \mathrm{~g}$ chez les chapons et de 33,37 g chez les mâles entiers n'a pas été significativement ( $>00,05)$ influencé par le chaponnage. Aucune mortalité n'a été observée après le chaponnage. Les résultats de cette étude confirment bien que le chaponnage peut permettre à l'éleveur de réaliser un bénéfice sur le plan de la vitesse de croissance et de la faible consommation d'aliments pour une bonne efficacité alimentaire par rapport aux mâles entiers.

(C) 2020 International Formulae Group. All rights reserved.

Mots clés : Aviculture, chapons, coquelet, efficacité alimentaire, mâles entiers.

\section{Effects of the caponnage on the zootechnical performances of local chicken in the city of Ngaoundere in Cameroon}

\begin{abstract}
Caponnage is an operation which consists in castrating and fattering a chicken (cockerel) in order to achieve greater mass, greater tenderness and therefore to improve the quality of its meat. The castrated cockerel is called capon: it is the fowl of festivals under other skies. Through the creation of added value on subjects considered to have no economic values, the caponnage can contribute to the development of cameroonian poultry
\end{abstract}


farming. In order to assess the effects of the caponnage on the zootechnical performances of local chickens, a study was carried out from 1st august to november 17th, 2018 in the peri-urban area of Ngaoundere. It involved 25 animals acquired from local breeders and divided into two batches. Batch 1 was made up of 14 subjects having undergone the caponnage and batch 2 made up of 11 whole males. The caponnage was carried out at 8 weeks of age and the two batches were fed ad libitum. Statistical analysis showed that the weight gain and the consumption index varied significantly $(\mathrm{p}<0,05)$ in capons compared to whole males. The same applies to the carcass yield and the weight of viscera. The weights of the heart, the liver and the gizzard are significantly $(\mathrm{p}<0,05)$ higher in capons than those of whole males. The abdominal fat, which was $40.18 \mathrm{~g}$ in capons and $33.37 \mathrm{~g}$ in whole males, was not significantly $(p>0,05)$ influenced by the caponnage. No mortality was observed after the caponnage. The results of this study confirm that caponnage can allow the breeder to make profit in terms of growth speed and low feed consumption for good feed efficiency compared to the whole males.

(C) 2020 International Formulae Group. All rights reserved.

Keywords: Aviculture, capons, cockerel, food efficiency, whole males.

\section{INTRODUCTION}

L'aviculture, du fait de ses nombreuses potentialités (courte durée du cycle de reproduction et de production, retour rapide sur les investissements, forte accessibilité à toutes les couches sociales, ...) occupe une place de choix dans la stratégie de lutte contre la pauvreté au Cameroun, renchérit par le document de stratégie pour la croissance et l'emploi (Dsce, 2010). Avec une contribution de $1 \%$ au PIB, le secteur avicole est un pilier de l'économie camerounaise avec 15 milliards de CFA et couvre $14 \%$ des besoins de la population en protéines animales (Minepia, 2013). Au Cameroun, le système d'élevage traditionnel ou villageois est dominé par la poule locale qui rassemble plus de $80 \%$ des effectifs totaux du pays (Tchoumboué, 2000).

Le développement de l'aviculture constitue un meilleur recours pour répondre à un besoin galopant de la population en protéines animales dans les courts délais. L'intérêt pour l'élevage des espèces à cycle court et particulièrement celui du poulet villageois se justifie d'une part, par la disponibilité de telles espèces dans le milieu rural, et d'autre part du fait que cet élevage n'impose ni une très grande technicité ni des coûts de production insupportables (Pym, 2013). La volaille locale reste la plus appréciée et la plus sollicitée des consommateurs par rapport aux poulets de souches importées (Pym, 2013). En dehors du mode d'abattage (saignée selon les exigences de la religion musulmane), il n'existe ni tabou, ni d'interdits pour la consommation du poulet et des œufs. Toutefois, l'usage des poulets élevés dans le mode villageois, obéit parfois aux normes culturelles qui soutiennent chaque tradition. Conscient d'une part que l'élevage des poules est quasi permanent dans l'économie familiale, et d'autre part que les poussins mâles, les coquelets et les coqs sont considérés comme gros mangeurs, agressifs, chair non appréciée et peu demandée sur le marché, non-valeurs économiques, la pratique du chaponnage (castration) pourrait contribuer à la résolution de ces problèmes. En effet, le chaponnage qui consiste en l'ablation totale des testicules du coq qui devient un chapon, permet à l'éleveur de revaloriser les coqs reconnus non qualifiés pour la reproduction et donc de faire une sélection aboutissant à l'amélioration de la race. Le chapon est ainsi engraissé pour améliorer la qualité de sa viande.

Au Cameroun, le chapon est un produit peu connu non seulement des consommateurs mais également des éleveurs. Il est de ce fait, quasiment absent du marché national. A notre connaissance, aucune étude n'a été entreprise sur la pratique du chaponnage sur le poulet local. C'est dans ce contexte que ce travail a été réalisé afin d'évaluer les effets du chaponnage sur les performances zootechniques du poulet local en déterminant la croissance pondérale, la consommation alimentaire, les caractéristiques de la carcasse, des viscères et le taux de mortalité. 


\section{MATERIEL ET METHODES \\ Zone d'étude}

L'étude s'est déroulée du 1 août au 17 novembre 2018 en zone périurbaine, dans le

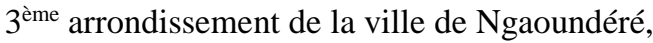
chef lieu de la région de l'Adamaoua. Elle couvre une superficie de $65000 \mathrm{~km}^{2}$ et se situe entre le $6^{\text {ème }}$ et le $8^{\text {ème }}$ degré de latitude Nord, le $10^{\text {ème }}$ et le $15^{\text {ème }}$ degré de longitude Est. Le climat est de type soudano-guinéen caractérisé par une saison de pluie d'avril à octobre avec un maximum de pluie en août, et une saison sèche de novembre à mars. La moyenne des précipitations annuelles est de $1500 \mathrm{~mm}$ et les températures moyennes oscillent entre $25^{\circ} \mathrm{C}$ et $45^{\circ} \mathrm{C}$. La population est estimée à environ 800000 habitants (Minepia, 2013). L'économie est basée sur l'élevage, l'agriculture, l'apiculture et le commerce.

\section{Echantillonnage}

Pour l'échantillonnage, cent deux œufs issus d'élevages traditionnels ont été collectés puis incubés durant 21 jours et 88 poussins ont été obtenus. Après le sexage et l'élevage des poussins pendant 60 jours, le tri des sujets à castrer reposait sur les critères individuels tels que le poids, l'âge, l'état général et la constitution des lots le jour de la castration s'est faite de manière aléatoire, à raison d'un lot 1 (témoin) de 11 mâles entiers et un lot 2 (expérimental) de 14 sujets à chaponner.

\section{Matériel animal}

Pour cette étude, 25 coquelets répartis en deux lots (lot 1 et lot 2) ont été utilisés à 7 semaines d'âge, donc une semaine avant le chaponnage.

\section{Conduite des animaux}

Dans cette expérimentation, la conduite des animaux s'est faite en deux phases dont la première allant de l'arrivée des poussins d'un jour à l'âge au chaponnage et qui a duré 8 semaines, puis la deuxième de 10 semaines comprise entre l'âge au chaponnage et à l'abattage.

Du démarrage à la croissance, les sujets étaient nourris ad libitum avec deux aliments (aliment chair-démarrage et aliment chair- croissance) achetés à la provenderie du 3ème arrondissement de Ngaoundéré qui fabrique les aliments de bétail et de volaille. Les aliments distribués aux poulets avaient une composition telle que présentée dans les Tableaux 1 et 2 .

$\mathrm{Du}$ chaponnage à l'abattage, le programme de prophylaxie des poulets de chair proposé par la Société des Provenderies du Cameroun (SPC) a été appliqué (Tableau 3). Le chaponnage a été effectué à la $8^{\text {ème }}$ semaine d'âge des coquelets et a duré 3 jours à raison de 4 à 5 animaux par jour. La méthode de chaponnage utilisée est celle décrite par Severin (2006) et cité par Kwin (2007) ainsi que M'kouar (2013). Trois jours avant l'opération, tous les animaux ont été mis sous antibiotique pour assurer la prévention d'éventuelles infections post-opératoires. Ils ont ensuite été mis sous diète hydrique de 12 heures avant l'opération. La contention a été faite en plaçant l'animal sur le côté sur une grille en le maintenant dans une position étendue à l'aide des bandes élastiques. Les ailes et les pattes sont étendues et attachées de manière à laisser la région costale bien visible (Photo 1).

Après avoir enlevé les plumes de la zone d'élection de l'opération, une incision cutanée musculaire a été faite au niveau du dernier espace intercostal ( $6^{\text {̀̀me }}$ et $7^{\text {ème }}$ côte), au niveau du tiers supérieur et du tiers moyen et doit être assez profonde pour bien exposer les sacs aériens (Photo 2).

Les testicules ont été repérés d'après leur localisation topographique, en arrière des poumons, près de l'extrémité antérieure des reins, le long de la paroi dorsale. L'exérèse des testicules s'est effectuée par torsion à l'aide de la pince à bout courbé. Le testicule est tenu au niveau de son pédoncule et tourné sur luimême puis tiré doucement vers l'extérieur jusqu'à la rupture du cordon testiculaire et de ses attaches (Photo 3). La Bétadine diluée à $10 \%$ a été utilisée pour aseptiser la plaie. Une suture au niveau de la peau en forme de $\mathrm{U}$ éversant à l'extrémité inférieure de l'incision a été réalisée et un antibiotique en spray (oxytetracycline) a été administré (Photo 4). L'opération a été répétée sur le côté opposé pour l'extraction du second testicule. 


\section{Collecte des données}

La collecte des données et la détermination des paramètres zootechniques ont été effectuées sur les animaux vivants, sur les carcasses et les viscères.

Sur les animaux vivants, les pesées hebdomadaires ont été effectuées sur chaque sujet à l'aide d'une balance électronique de précision $1 \mathrm{~g}$ qui était toujours tarée avant chaque prise (Photo 5). Elles ont permis d'évaluer l'évolution pondérale des poulets et le gain moyen hebdomadaire $(\mathrm{GMH})$. Les quantités d'aliments distribués dans les différents lots et les refus ont été pesées quotidiennement pour déterminer les quantités consommées par lot.

Sur les animaux abattus, le poids carcasse a été mesuré après éviscération complète de l'animal. A l'aide d'une règle graduée au millimètre, la taille de la crête et des barbillons a été mesurée.

Sur les viscères, les poids du cœur, du foie et du gésier ont été pesés à l'aide d'une balance électronique.

Les données collectées ont permis de calculer les paramètres zootechniques que sont le poids vif (PV), le gain moyen hebdomadaire (GMH), l'indice de consommation (IC), le rendement carcasse (RC) et le taux de mortalité (TM).

\section{Analyse statistique}

Le tableur Excel ${ }^{\circledR}$ a été utilisé pour la saisie des données. Le calcul des moyennes, des écarts-types, des variances et la comparaison des moyennes (Test de Student) ont été réalisés à l'aide du logiciel Statgraphics Centurion Version 17.2. Une valeur de $\mathrm{p}<0,05$ a été considérée comme significative.

Tableau 1 : Valeur alimentaire des aliments distribués aux poussins.

\begin{tabular}{lcccccc}
\hline Age (semaines) & EM (kcal/kg) & PB (\%) & Ca (\%) & P (\%) & Met (\%) & Lys (\%) \\
\hline $0-3$ & 2800 & & 22,54 & 1,4 & 0,46 & \\
0,41 & & & 1,32 & & & \\
$>3$ & 2800 & 19,65 & 1,16 & 0,43 & 0,37 & 1,19 \\
\hline
\end{tabular}

$\mathrm{EM}=$ Energie Métabolisable, $\mathrm{PB}=$ Protéines Brutes, $\mathrm{Ca}=$ Calcium, $\mathrm{P}=$ Phosphore, Met= Méthionine, Lys=Lysine

Tableau 2 : Composition de la ration expérimentale des deux lots.

\begin{tabular}{lcc}
\hline Composition (\%) & Aliment chair démarrage & Aliment chair Croissance \\
\hline Matières sèches & 92,76 & 93,62 \\
Matières minérales & 6,88 & 6,25 \\
Protéines brutes & 21,19 & 18,46 \\
Matières grasses & 4,39 & 6,14 \\
Cellulose brute & 11,20 & 8,91 \\
Calcium & 0,70 & 0,62 \\
Phosphore & 0,65 & 0,47 \\
\hline
\end{tabular}


Tableau 3 : Programme de prophylaxie appliquée dans cette étude.

\begin{tabular}{llc}
\hline Période (jours) & Interventions & Produits utilisés \\
\hline J $54-$ J57 & Antibiothérapie & Trisulmicyne \\
J $58-$ J60 & Vitaminothérapie & Amintotal \\
J $63-$ J65 & Déparasitage & Piperazine \\
J66 - J70 & Oligo-éléments & oligophos \\
J $80-$ J83 & Anticoccidien & Anticox \\
J $90-$ J93 & Déparasitage & Piperazine \\
J115 - J118 & Vitaminothérapie & Amintotal \\
\hline J= Jour & &
\end{tabular}

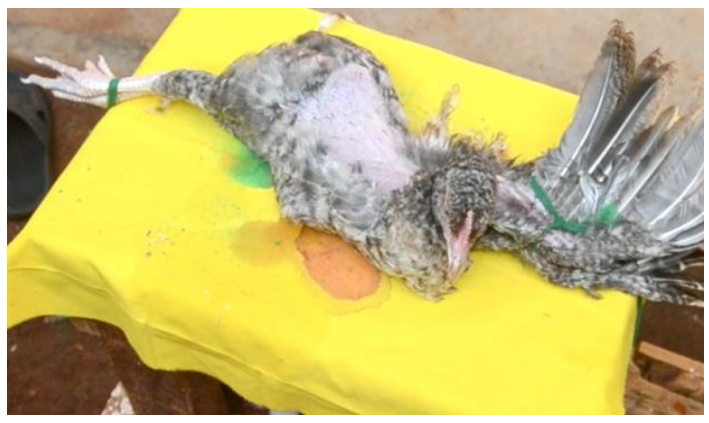

Photo 1 : Technique de contention du coquelet et de mise en évidence de la zone d'élection.

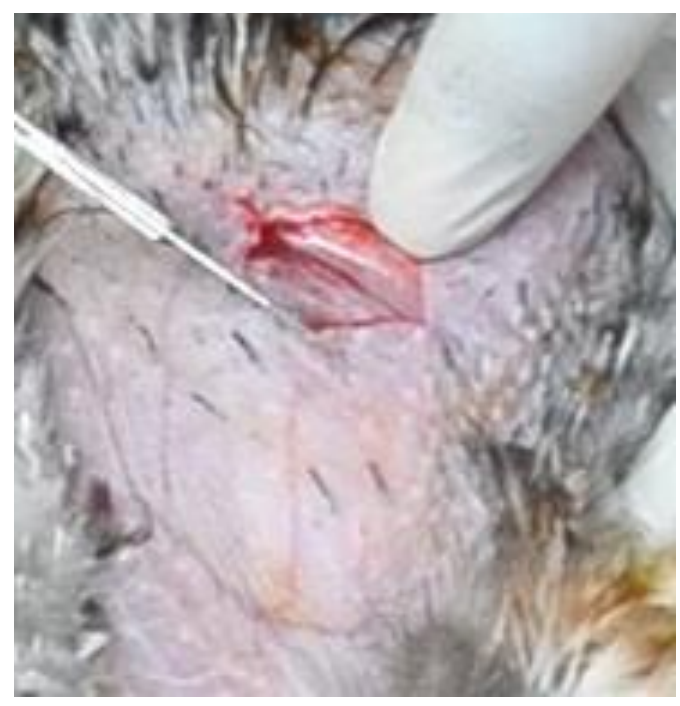

a

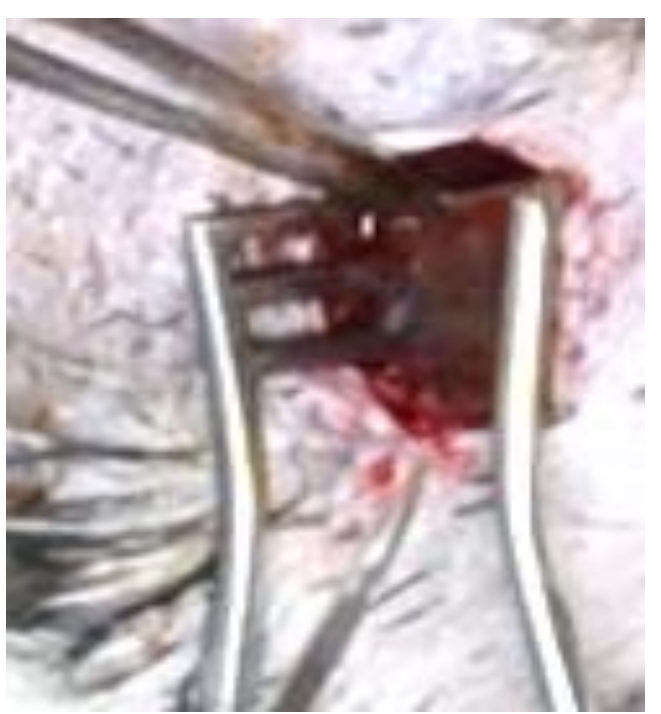

b

Photo 2 : Incision cutanée (Photo 2a) et mise en évidence du sac aérien (Photo 2b). 


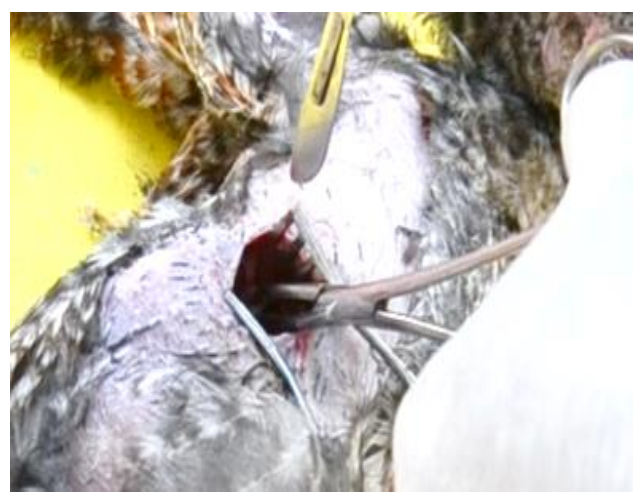

a
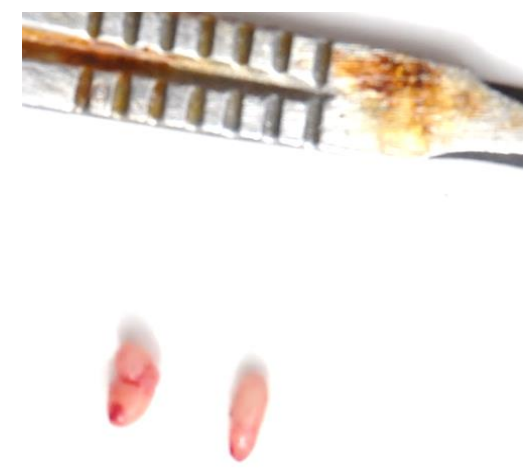

b

Photo 3 : Exérèse des testicules (Photo 3a) et testicules extraits (Photo 3b).

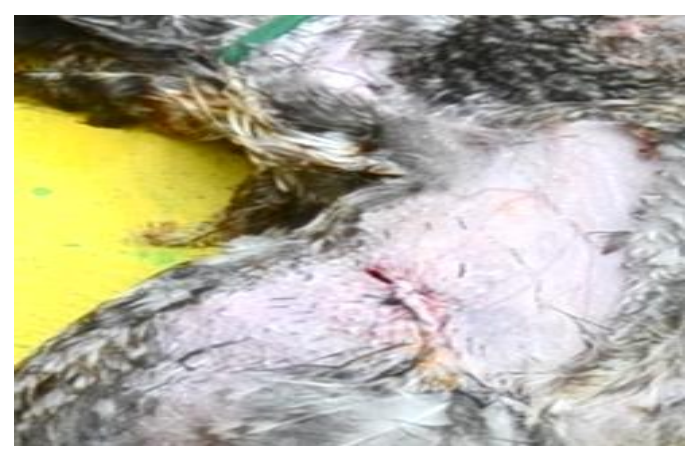

a

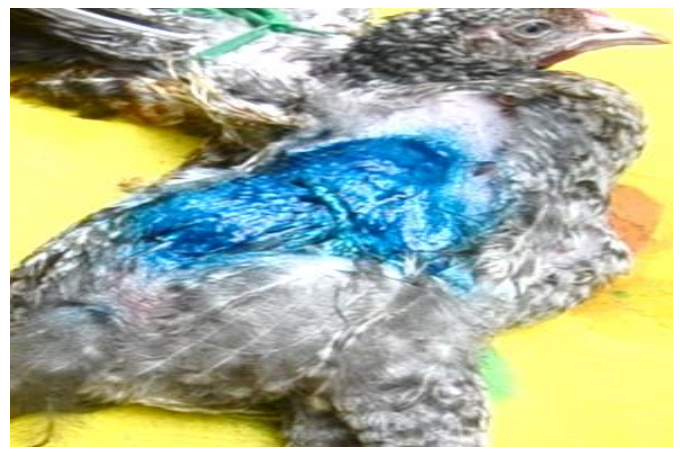

b

Photo 4 : Suture de la plaie (Photo 4a) et administration du cicatrisant (Photo 4b)

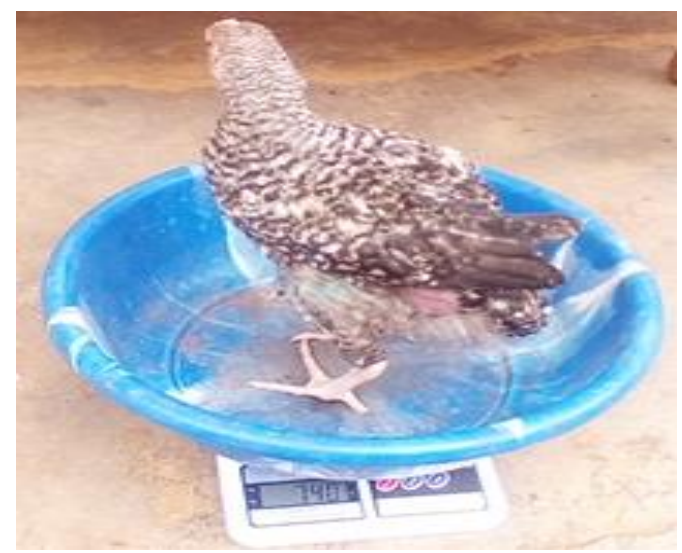

Photo 5 : Évaluation du poids du poulet. 


\section{RESULTATS}

Effets du chaponnage sur les performances de croissance

\section{Sur la croissance pondérale}

La Figure 1 illustre l'évolution de la croissance pondérale des poulets.

Il ressort de l'analyse de cette figure qu'au terme de 7 semaines d'expérimentation, la croissance pondérale a augmenté de manière régulière aussi bien chez les chapons que chez les coquelets entiers. Cette croissance était significativement $(\mathrm{p}<0,05)$ plus élevée chez les chapons comparativement aux coquelets entiers. La supériorité pondérale du lot des castrés se maintient jusqu'en fin d'expérimentation.

\section{Sur le gain moyen hebdomadaire (GMH)}

L'évolution du gain moyen hebdomadaire $(\mathrm{GMH})$ des poulets est représentée dans le Tableau 4.

Il ressort de ce tableau que pendant la $1^{\text {ère }}$ semaine de l'expérimentation, la croissance des chapons était plus lente que celle des mâles entiers. La situation s'est inversée dans la $3^{\text {ème }}$ moitié de l'essai et était significativement $(\mathrm{p}<0,05)$ plus élevée chez les chapons pendant les semaines 3, 4, 5 et 7 par rapport aux mâles entiers. A la $6^{\text {ème }}$ semaine, le GMH était significativement $(\mathrm{p}<0,05)$ plus élevée chez les mâles entiers que chez les chapons.

Effets du chaponnage sur la consommation et l'indice de consommation alimentaires Sur la consommation alimentaire

Les résultats sur la consommation alimentaire sont présentés dans le Tableau 5.

Il ressort de ce tableau que la consommation alimentaire était significativement $(\mathrm{p}<0,05)$ plus élevée chez les mâles entiers à partir de la $5^{\text {ème }}$ semaine jusqu'à la $7^{\text {ème }}$ semaine par rapport aux chapons.

\section{Sur l'indice de consommation (IC) alimentaire \\ Les résultats sur les variations de l'indice de consommation en fonction des semaines sont présentés dans le Tableau 6. \\ Il ressort de ce tableau que l'indice de consommation (IC) était significativement}

$(\mathrm{p}<0,05)$ plus élevé chez les mâles entiers que chez les chapons. La moyenne de l'IC à la fin de l'expérimentation était significativement $(\mathrm{p}<0,05)$ plus élevée chez les mâles entiers $(11,75)$ que chez les chapons $(6,25)$. Globalement, les chapons ont une moins bonne efficacité alimentaire que les mâles entiers.

Effet du chaponnage sur les caractéristiques de la carcasse et des organes Sur la conformation externe de la carcasse

La Photo 6 montre l'effet du chaponnage sur la conformation des muscles de la poitrine (bréchet) des chapons (Photo 6a) et des mâles entiers (Photo 6b).

Il ressort de l'observation de ces photos que le muscle de la poitrine (bréchet) des chapons est significativement $(\mathrm{p}<0,05)$ plus charnue et convexe que celui des mâles entiers. De même, les muscles de la cuisse sont plus développés chez les chapons comparativement aux mâles entiers.

\section{Sur la carcasse et quelques organes}

Les résultats de l'effet du chaponnage sur les caractéristiques de la carcasse et des organes sont présentés dans le Tableau 7.

Il ressort de ce tableau que le poids carcasse, le poids vif et le rendement carcasses ont plus élevés chez les chapons que chez les mâles entiers mais cette différence n'est pas significative $(p>0,05)$. Le poids du gras abdominal est plus élevé chez les chapons $(40,18 \pm 12,50 \mathrm{~g})$ que chez les mâles entiers (33, $37 \pm 98,50 \mathrm{~g}$ ). Les poids du cœur, du foie et du gésier sont significativement $(\mathrm{p}<0,05)$ plus élevés chez les chapons que ceux des mâles entiers.

\section{Effet du chaponnage sur le développement} des appendices

A la fin de l'expérimentation, les chapons ont présenté des crêtes et des barbillons décolorés et moins développés que les mâles entiers (Tableau 8).

\section{Effet du chaponnage sur la mortalité}

Au terme de notre expérimentation, le taux de mortalité était de $0 \%$ chez les chapons et les mâles entiers. Aucun chapon n'a succombé à la suite au chaponnage. 


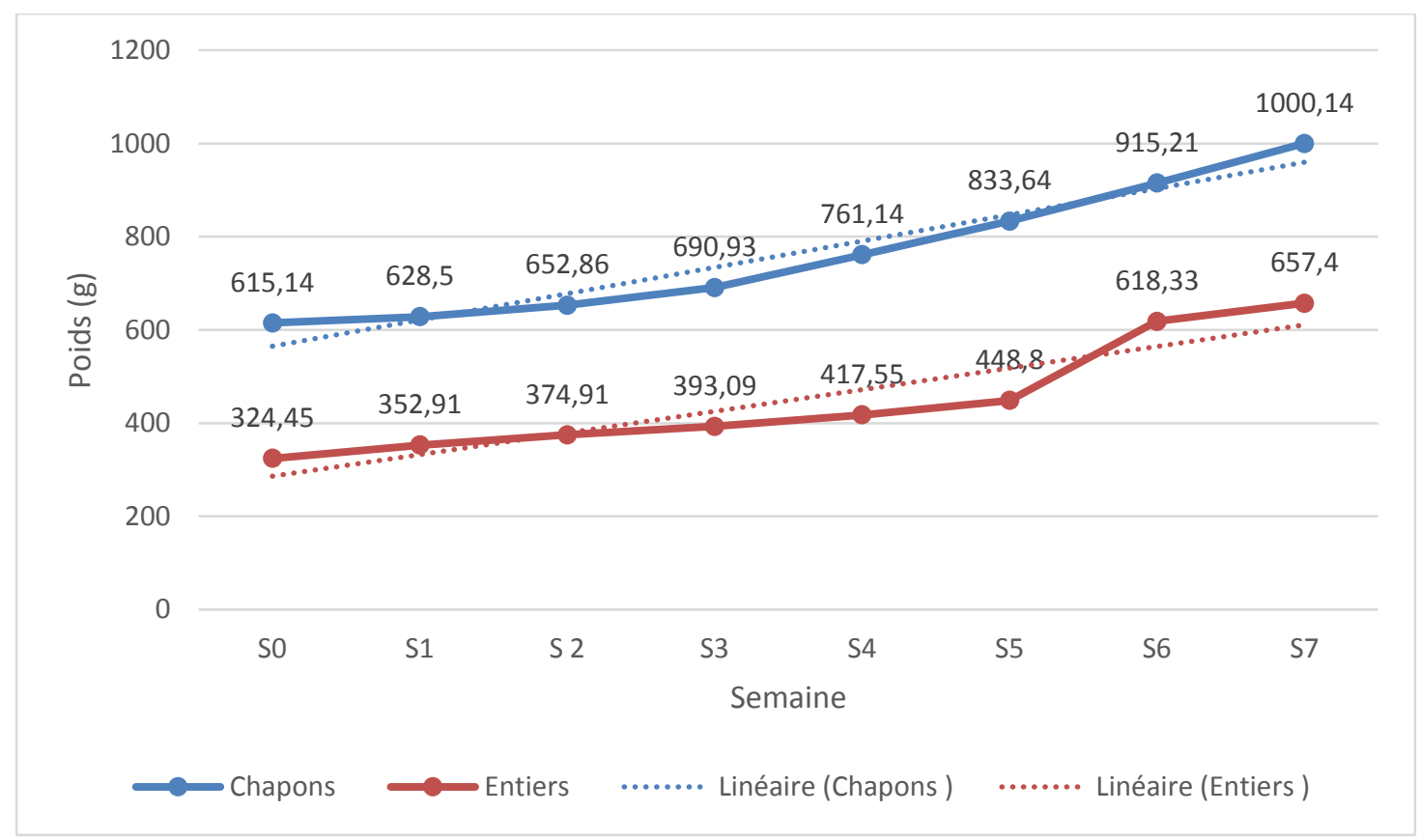

Figure 1 : Evolution du poids des chapons et des mâles entiers en fonction de l'âge.

Tableau 4: Effets du chaponnage sur le gain moyen hebdomadaire.

\begin{tabular}{llllc}
\hline & Temps & Chapons $(\mathbf{g})$ & Entiers $(\mathbf{g})$ & Valeur de p \\
\hline & S1 & $13,36 \pm 7,71$ & $28,45 \pm 6,25$ & 0,00 \\
G.M.H (g) & S2 & $24,36 \pm 7,06$ & $22,00 \pm 8,47$ & 0,00 \\
& S3 & $38,07 \pm 33,30$ & $18,18 \pm 10,36$ & 0,00 \\
& S4 & $70,21 \pm 20,83$ & $24,45 \pm 19,63$ & 0,00 \\
& S5 & $72,50 \pm 2,76$ & $31,25 \pm 23,89$ & 0,00 \\
& S6 & $81,57 \pm 81,67$ & $169,53 \pm 74,86$ & 0,00 \\
& S7 & $84,93 \pm 36,09$ & $39,07 \pm 84,45$ & 0,00 \\
\hline
\end{tabular}

$\mathrm{S}:$ Semaine, GMH= Gain moyen hebdomadaire.

Tableau 5: Effets du chaponnage sur la consommation alimentaire individuelle par semaine.

\begin{tabular}{llllc}
\hline & Temps & Chapons $(\mathbf{g})$ & Entiers $(\mathbf{g})$ & Valeur de $\mathbf{p}$ \\
\hline & S1 & $26,62 \pm 3,84$ & $29,20 \pm 2,47$ & 0,16 \\
& S2 & $27,13 \pm 4,40$ & $28,51 \pm 0,78$ & 0,53 \\
QACIS & S/s & $29,29 \pm 1,88$ & $29,24 \pm 0,50$ & 0,94 \\
& S4 & $31,33 \pm 2,17$ & $30,95 \pm 1,05$ & 0,68 \\
& S5 & $37,79 \pm 6,91$ & $47,95 \pm 1,75$ & 0,00 \\
& S6 & $48,37 \pm 2,25$ & $49,34 \pm 1,18$ & 0,54 \\
& S7 & $49,29 \pm 2,10$ & $53,94 \pm 1,92$ & 0,00 \\
\hline
\end{tabular}

$\mathrm{S}=$ Semaine, $\mathrm{QACIS}=$ Quantité d'aliment consommée individuellement par semaine. 
Tableau 6 : Effet du chaponnage sur l'indice de consommation alimentaire.

\begin{tabular}{clllc}
\hline & Temps & Chapons (g) & Entiers (g) & Valeur de p \\
\hline & S1 & $13,95 \pm 0,50$ & $13,11 \pm 0,40$ & 0,00 \\
& S2 & $7,80 \pm 0,62$ & $14,11 \pm 0,09$ & 0,00 \\
IC & S3 & $5,80 \pm 0,06$ & $17,51 \pm 0,05$ & 0,00 \\
& S4 & $3,40 \pm 0,10$ & $13,78 \pm 0,05$ & 0,00 \\
& S5 & $3,93 \pm 2,50$ & $10,74 \pm 0,07$ & 0,00 \\
& S6 & $4,47 \pm 0,03$ & $4,23 \pm 0,02$ & 0,00 \\
& S7 & $4,37 \pm 0,06$ & $8,86 \pm 0,02$ & 0,00 \\
\hline
\end{tabular}

$\mathrm{S}=$ Semaine, $\mathrm{IC}=$ Indice de consommation

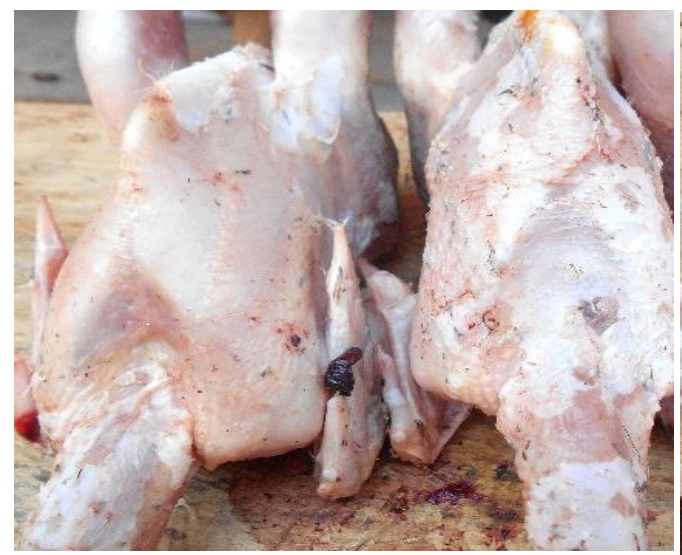

a

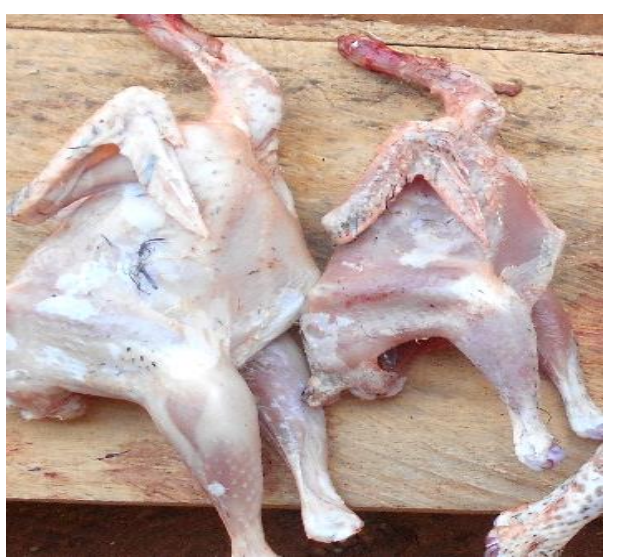

b

Photo 6: Conformation externe du bréchet et des cuisses (Photo $6 \mathrm{a}=$ chapons, Photo $6 \mathrm{~b}=$ mâles entiers).

Tableau 7 : Effet du chaponnage sur les caractéristiques de la carcasse et des organes.

\begin{tabular}{lccc}
\hline Caractéristiques & Chapons & Mâles entiers & Valeur de p \\
\hline Poids carcasse $(\mathrm{g})$ & $651,60 \pm 120,42$ & $352,25 \pm 100,13$ & 0,00 \\
Poids vif $(\mathrm{g})$ & $1025,40 \pm 180,63$ & $590,50 \pm 165,56$ & 0,00 \\
Rendement carcasse (\%) & $63,58 \pm 1,23$ & $47,67 \pm 0,76$ & 0,00 \\
Poids du gras abdominal (g) & $40,18 \pm 12,50$ & $33,37 \pm 98,50$ & 0,00 \\
Poids des organes $(\mathrm{g}):$ & & & \\
- $\quad$ Cour & $6,00 \pm 1,58$ & $3,50 \pm 0,580,00$ & \\
- Foie & $22,4 \pm 4,04$ & $15,25 \pm 2,63$ & 0,03 \\
$-\quad$ Gésier & $27,80 \pm 3,03$ & $19,00 \pm 5,290,01$ & \\
\hline
\end{tabular}

Tableau 8 : Effet du chaponnage sur le développement des appendices.

\begin{tabular}{lccc}
\hline Caractéristiques & Chapons & Mâles entiers & Valeur de $\mathbf{p}$ \\
\hline Crêtes $(\mathrm{cm})$ & 3,46 & 4,14 & 0,290 \\
Barbillons $(\mathrm{cm})$ & 2,82 & 3,17 & 0,220 \\
\hline
\end{tabular}




\section{DISCUSSION}

Au travers de cette expérimentation, il est important de constater que la croissance pondérale et le gain moyen hebdomadaire $(\mathrm{GMH})$ étaient significativement plus élevés chez les chapons par rapport aux mâles entiers. Cette supériorité a été observée de manière croissante et régulière jusqu'à la fin de l'expérimentation contrairement au GMH des mâles entiers qui a évolué en dents de scie. Ce résultat est en accord avec les travaux de Zawacka (2016) qui ont rapporté un résultat similaire en chaponnant des Green-legged Partridge. Il est en désaccord avec celui de Kwin (2007) qui avait castré des coquelets de race Leghorn blanche à Dakar au Sénégal et Mahmud (2013) qui n'a pas noté de différence significative 8 semaines après la castration des coquelets au Nigéria. La supériorité du gain de poids chez les chapons serait due à l'effet de la castration qui aurait rendu ces derniers moins actifs, c'est-à-dire moins mobiles, moins fouilleurs, moins agressifs, plus dociles avec un tempérament calme que les mâles entiers. Toutefois, le GMH a été faible chez le chapon à la $1^{\text {ère }}$ semaine. Cette contreperformance pourrait découler de l'effet traumatisant de la castration et de son impact sur l'état général de l'animal. Etant donné qu'il s'agissait de race locale à croissance lente, le gain de poids sur la durée de 8 semaines était faible comparé à celui des souches lourdes. La faible consommation alimentaire observée chez les chapons $(1856,53 \mathrm{~g})$ contre $2365,80 \mathrm{~g}$ chez les mâles entiers est contraire aux résultats rapportés par Mahmud (2013) et Lindell (2015). Ceci peut s'expliquer par le fait qu'après la $1^{\text {ère }}$ semaine post chirurgicale, la faible consommation d'aliments est certainement liée à la convalescence. La faible consommation durant la suite de l'expérimentation pourrait également s'expliquer par le fait que le tempérament plus calme des chapons diminue leurs besoins en énergie d'entretien ce qui influence négativement les besoins énergétiques totaux, et par conséquent entraîne la sous consommation alimentaire.

Le rendement carcasse est significativement élevé chez les chapons. Ce résultat corrobore avec ceux de Cheng (2012) à
Taïwan, de Getu (2014) en Ethiopie, de Calik (2015) et de Kwiecien (2015) mais est en désaccord avec ceux rapportés par Kwin (2007) et Mahmud (2013). Cette différence pourrait être liée à la race. Dans notre étude, la race locale (poulet villageois), de poids léger, de faible performance a été utilisée contre des races mi- lourdes ou lourdes ou même améliorées.

L'augmentation non significative de poids du gras abdominal chez les chapons par rapport aux mâles entiers est en adéquation avec la moins bonne efficacité alimentaire des chapons et traduit une tendance à l'obésité unanimement reconnue chez les animaux castrés (Brandsdetter, 2000). Elle pourrait s'accompagner de dépôt accru de gras intramusculaire qui peut justifier l'appréciation de la qualité des chapons par les consommateurs.

S'agissant des organes, le développement du cœur et du foie chez les chapons par rapport aux mâles entiers est influencé par les androgènes. Ces résultats sont en désaccord avec ceux de Cheng (2012) et Lindell (2015) qui ont obtenu des poids plus élevés chez les mâles entiers. Le gésier a un poids plus élevé chez les chapons par rapport aux mâles entiers; ceci est en accord avec les travaux de Lindell (2015).

La faible taille des appendices chez les chapons par rapport aux mâles entiers confirme les résultats rapportés par Syméon (2012). Cela serait sans doute dû au fait que les androgènes intervenant dans le développement de la crête et des barbillons font défaut chez les chapons à la suite de leur castration.

Dans cette étude, aucune mortalité n'a été observée contrairement aux résultats de Kwin (2007) qui a rapporté un taux de mortalité de $6,6 \%$ chez les chapons et de Tor (2002) un taux de mortalité de $1 \%$. Ceci pourrait s'expliquer d'une part par la maîtrise de la technique du chaponnage, et d'autre part par la race utilisée, le poulet local (villageois) de souche légère, assez rustique, plus résistant au chaponnage et que les mesures de prophylaxie ont été bien respectées. 


\section{Conclusion}

Au terme de cette étude qui a porté sur l'évaluation des effets du chaponnage sur les performances zootechniques chez le poulet local dans la ville de Ngaoundéré au Cameroun, les résultats ont montré que la vitesse de croissance et le gain de poids des chapons étaient significativement $(p>0,05)$ plus élevés que ceux des mâles entiers. La consommation alimentaire était plus élevée chez les mâles entiers pour une efficacité alimentaire faible comparée aux chapons. Le rendement carcasse était significativement $(\mathrm{p}>0,05)$ plus élevé chez chapons par rapport aux mâles entiers. De même, les poids du cœur, $\mathrm{du}$ foie et du gésier étaient significativement ( $p>0,05)$ plus élevés chez les chapons que ceux des mâles entiers. Le bénéfice que peut générer cette pratique ne saurait être évalué avec précision, car la valeur du chapon n'est pas connue de la population. Néanmoins, le chaponnage a permis de réaliser une économie sur la quantité d'aliments distribuée aux chapons et aussi sur la durée d'engraissement au vue du gain de poids meilleur chez les chapons. Ainsi, le chaponnage peut donc constituer une alternative pour la sélection des reproducteurs en castrant tous les coqs qui ne seront pas utilisés pour la reproduction. Le chaponnage peut être également une source d'emplois. En perspective, il serait nécessaire que des études complémentaires soient menées, notamment sur les qualités, organoleptiques de la viande du chapon issu du poulet local, sur l'évaluation de la rentabilité financière d'une telle activité et sur le sexage précoce de la population de poulet local.

\section{CONFLIT D'INTERETS}

Les auteurs ne déclarent aucun conflit d'intérêts.

\section{CONTRIBUTIONS DES AUTEURS}

Tous les auteurs ont contribué à la prise en charge des matériels didactique, technique et à la rédaction du manuscrit. Tous les auteurs ont lu et approuvé la version finale du manuscrit.

\section{REMERCIEMENTS}

Les auteurs expriment leurs sincères remerciements aux vétérinaires et éleveurs de volaille de la commune de Ngaoundéré $3^{\text {ème}}$, pour leur aide précieuse sur le terrain. Nos remerciements s'adressent également à la société de provenderie de Ngaoundéré $3^{3 \text { ème }}$ pour la fourniture en aliments malgré un contexte difficile, et particulièrement à $\mathrm{M}$. NDOBGA Marcel qui a veillé sur les animaux durant toute l'expérimentation.

\section{REFERENCES}

Brandstetter A, Pfaffl MN, Hocquette JF, Sauerwein H. 2000. Effects of muscle type, castration, age and compensatory growth rate on androgen receptor mRNA expression in bovin skeletal muscle. $J$. Anim. Sci., 78(3): 629-637. DOI: https://doi.org/10.2527/2000.783629x

Calik J. 2015. Effects of caponizing Yellow leg Partridge (Z-33) cockerels on body weight And meat quality. Acta. Sci. Pol. Zootechnica, 14(1): 51-60. DOI: https://doi.org/10.1515/aoas.2015.0002

Cason JA, Fletcher DL, Burke WH. 1998. Effects of caponization on broiler growth.

Poult Sci., 67(6): 979-981. DOI: https://doi.org/10.3382/ps.0670979

Cheng YL, Jeun CH, Tien CW. 2012. Effects of age and caponization on blood Parameters and bone development of male native chickens in Taiwan. Asian. Aust. J. Anim. Sci., 25 (7): 994-1002. DOI :

https://doi.org/10.5713/ajas.2011.11210

Dsce. 2009. Document de stratégie pour la croissance et l'emploi : revue des grands projets au Cameroun, $167 \mathrm{p}$.

Fennell MJ, Scanes CG, Clin G. 1992. Inhibition of growth in chickens by testosterone. Poult. Sci., 71: 357-366. DOI : https//doi.org/10.3382/ps.0710357

Getu A. 2014. A phenotypic and genetic characterized indigenous chicken ecotypes in Ethiopia. International Journal of Genetics, 4 (1): 22-27. DOI: https://doi.org/10.5829/idosi.ijg.2014.4.1 .8523 
Hamidou O. 1985. L'aviculture en République du Cameroun. Thèse Doc. Méd. Vét., 4: $102 p$.

Kemp JD, Crouse JD, Deweese W, Moody WG. 1970. Effect of slaughter weight and castration on carcass characteristics of lambs. J. Anim. Sci., 30 (3): 348-354.

DOI:

https://doi.org/10.2527/jas.1970.373348x

Kwiecien M, Kasperek K, Grela E. 2015. Effect of caponzation on the production performance, slaughter yield and fatty acid profile of muscles of Green leg Partridge cocks. Journal of Food Science and Food Technology, 52 (11): 72277235.

DOI :

https://doi.org/10.1007/s13197.015.1856 .6

Kwin H. 2007. Effet du chaponnage sur les performances de croissance et les caractéristiques de carcasse des coquelets. Th. Méd. Vét., 30: 82p.

Lindell J. 2015. Local poultry production in rural Zambia and the effect of light on poultry performance. Swedish University of Agricultural Sciences, Faculty of Veterinary Medicine and Animal Science, Department of Animal Nutrition and Management, 36p.

Mahmud M, Shaba P, Yisa H, Gana J, Ndagimba R. 2013. Effects of surgical caponisation on Growth, carcass and some haematological parameters in cockerel chickens. Sokoto Journal of Veterinary Science, 11 (2): 57-62. DOI: https://doi.org/10.4314/sokjvs.v11i2.9

Mankor A. 2009. Evolution du secteur de l'élevage ouest-africain, consommation urbaine des viandes en Afrique de l'ouest: l'exemple de Dakar. Grain de Sel, 46-47.

Minepia. 2013. Direction des Services Vétérinaires. Rapport annuel du Service Régional de la Protection Sanitaire de l'Adamaoua, Ngaoundéré, 320p.
M'kouar F, Bouzouaya M. 2013. Le chaponnage chirurgical des volailles en Tunisie, $\mathrm{N}^{\circ} 27,7 \mathrm{p}$.

Pym R. 2013. Poultry genetics and breeding in developing countries, contribution of indigenous genotypes to production and consumption of poultry meat and eggs. Food and Agriculture Organization of United Nations. Poultry Development Review, $127 \mathrm{p}$.

Severin K, Masek T, Janicki Z, Konjevic D, Slavica A. 2006. Caponisation of pheasants at different age. Vet. Arhiv., 76: 211-219.

Symeon G, Mantis F, Rogdakis E. 1992. Effects of caponization on growth performance, carcass composition and meat quality of males of a layer line. Animal, 6: 2023-2030. DOI: https://doi.org/10.1017/51751731112001 024

Tchoumboué J, Manjeli Y, Teguia A, Ewane NJ. 2000. Productivité et effets comparés de trois systèmes de conduite de l'élevage sur les performances de l'aviculture villageoise dans les hautes terres de l'Ouest-Cameroun. Science Agronomique et Développement, 2(1): 614.

Tor M, Estany J, Francesch A, Cubilo MD. 2002. Comparaison de la composition lipidique de la viande, des tissus adipeux et des muscles de coqs et de chapons. Anim. Res., 54: 413-424. DOI: https://doi.org/10.1051/animres:2002035

Zawacka M, Murawska D, Gesek M. 2016. The effect of age and castration on the growth rate, blood lipid profile, liver histology and feed conversion in Green legged Partridge

cockerels and capons. Animal, 11(6): 1017-1026. DOI: https://doi.org/10.1017/51571731116002 378 